\title{
On a multiplicative hybrid problem
}

\author{
by
}

\author{
WEN-GUANG ZHAI (Jinan)
}

1. Main results. In [2], H. Iwaniec and A. Sárközy considered the following multiplicative hybrid problem. Let $N$ be a natural number large enough, $G_{1}$ and $G_{2}$ be subsets of $\{N+1, \ldots, 2 N\},\left|G_{1}\right| \gg N,\left|G_{2}\right| \gg N$. They proved that there exist integers $n_{1}, n_{2}, b$ with $n_{1} \in G_{1}, n_{2} \in G_{2}$, and

$$
n_{1} n_{2}=b^{2}+O\left(b^{1 / 2} \log ^{1 / 2} b\right) \text {. }
$$

In this paper, we consider a more general case. Let $k \geq 3$ be a fixed integer and $N$ be an integer large enough. $G_{1}, \ldots, G_{k}$ are $k$ subsets of $\{N+$ $1, \ldots, 2 N\}$. Suppose $\Delta$ is a real number satisfying $0<\Delta \leq 1 / 2$. Let $S_{k}$ denote the number of solutions to the inequality

$$
\left\|\left(n_{1} \ldots n_{k}\right)^{1 / k}\right\| \leq \Delta, \quad n_{1} \in G_{1}, \ldots, n_{k} \in G_{k} .
$$

We shall estimate $S_{k}$. Our main result is

THEOREM 1. If $T_{k}=\left|G_{1}\right| \ldots\left|G_{k}\right|$, then

$$
S_{k}=2 \Delta T_{k}+O\left(T_{k}^{1 / 2} N^{(k-1) / 2} \log (k-2) / 2 N\right)
$$

for $k \geq 4$ and

$$
S_{3}=2 \Delta T_{3}+O\left(N^{5 / 2} \log ^{1 / 2} N\right) .
$$

The constant implied in (2) depends on $k$.

As an application of Theorem 1 we have immediately

THEOREM 2. If $\left|G_{1}\right| \gg N, \ldots,\left|G_{k}\right| \gg N$, then there exist integers $n_{1}, \ldots, n_{k}, b$ with $n_{1} \in G_{1}, \ldots, n_{k} \in G_{k}$ such that

$$
n_{1} \ldots n_{k}=b^{k}+O\left(b^{k-3 / 2} \log ^{(k-2) / 2} b\right) \quad(k \geq 3) .
$$

The constant implied in (4) depends on $k$.

Remark. Theorem 2 of [1] implies our Theorem 2 with a weak log factor in the error term for $k \geq 4$. So we use a little different method to get a slightly better result. 
Notations. Throughout this paper, $\|x\|=\min \{|x-n| \mid n$ is an integer $\}$, $\{x\}$ means the fractional part of $x, b(x)=\{x\}-1 / 2$ and $e(x)=\exp (2 \pi i x)$. $|G|$ stands for the number of elements of $G$. As usual, $d_{t}(n)$ denotes the number of ways $n$ can be written as a product of $t$ factors.

2. Some lemmas. To complete our proof we need some lemmas.

Lemma 1 (Lemma 1 of [2]). Let $A$ and $B$ be two finite sets of real numbers, $A \subset[-X, X], B \subset[-Y, Y]$. Then for any complex functions $u(x)$ and $v(y)$, we have

$$
\begin{aligned}
& \left|\sum_{x \in A} \sum_{y \in B} u(x) v(y) e(x y)\right|^{2} \\
& \leq 2 \pi^{2}(1+X Y) \sum_{x \in A} \sum_{\substack{x^{\prime} \in A \\
2 Y\left|x-x^{\prime}\right| \leq 1}}\left|u(x) u\left(x^{\prime}\right)\right| \sum_{y \in B} \sum_{\substack{y^{\prime} \in B \\
2 X\left|y-y^{\prime}\right| \leq 1}}\left|v(y) v\left(y^{\prime}\right)\right| .
\end{aligned}
$$

Lemma 2 (Theorem 12.2 of [3]). Suppose $t \geq 2$ is an integer and write

$$
D_{t}(x)=\sum_{n \leq x} d_{t}(n)=x P_{t}(\log x)+\Delta_{t}(x),
$$

where $P_{t}(u)$ is a polynomial of degree $t-1$ in $u$. Then $\Delta_{t}(x)=O\left(x^{\theta_{t}}\right)$ with $\theta_{t}=(t-1) /(t+1)$.

Lemma 3. Suppose $t$ is an integer, $2 \leq t \leq k, Q_{1}, \ldots, Q_{t}$ are $t$ subsets of $\{N+1, \ldots, 2 N\}$, $\delta$ is a real number, $0<\delta<1$. Let $A\left(Q_{1}, \ldots, Q_{t} ; \delta\right)$ be the number of solutions to the inequality

$$
\left|\left(n_{1} \ldots n_{t}\right)^{1 / k}-\left(\bar{n}_{1} \ldots \bar{n}_{t}\right)^{1 / k}\right| \leq \delta, \quad n_{i}, \bar{n}_{i} \in Q_{i}, i=1, \ldots, t .
$$

Then

$$
A\left(Q_{1}, \ldots, Q_{t} ; \delta\right) \ll\left(N^{t \theta_{t}}+\delta N^{t(k-1) / k} \log ^{k-1} N\right)\left|Q_{1}\right| \ldots\left|Q_{t}\right| .
$$

Proof. The inequality (5) implies

(6) $\left|\left(n_{1} \ldots n_{t}\right)-\left(\bar{n}_{1} \ldots \bar{n}_{t}\right)\right| \leq \delta k(2 N)^{t(k-1) / k}, \quad n_{i}, \bar{n}_{i} \in Q_{i}, i=1, \ldots, t$.

For any fixed $\left(\bar{n}_{1}, \ldots, \bar{n}_{t}\right)$, the number of solutions of $(6)$ is

$$
\begin{aligned}
& S\left(\bar{n}_{1}, \ldots, \bar{n}_{t}\right) \\
& \quad \leq D_{t}\left(\bar{n}_{1} \ldots \bar{n}_{t}+\delta k(2 N)^{t(k-1) / k}\right)-D_{t}\left(\bar{n}_{1} \ldots \bar{n}_{t}-\delta k(2 N)^{t(k-1) / k}\right) .
\end{aligned}
$$

For simplicity, we put $x_{0}=\bar{n}_{1} \ldots \bar{n}_{t}, y_{0}=\delta k(2 N)^{t(k-1) / k}$. Then by Lemma 2 we get 
(8) $S\left(\bar{n}_{1}, \ldots, \bar{n}_{t}\right)$

$$
\begin{aligned}
& \ll\left(x_{0}+y_{0}\right) P_{t}\left(\log \left(x_{0}+y_{0}\right)\right)-\left(x_{0}-y_{0}\right) P_{t}\left(\log \left(x_{0}-y_{0}\right)\right)+O\left(\left(x_{0}+y_{0}\right)^{\theta_{t}}\right) \\
& =2 y_{0}\left(P_{t}\left(\log x^{\prime}\right)+P_{t}^{\prime}\left(\log x^{\prime}\right)\right)+O\left(\left(x_{0}+y_{0}\right) \theta_{t}\right) \\
& \ll \delta N^{t(k-1) / k} \log ^{t-1} N+N^{t \theta_{t}} .
\end{aligned}
$$

So we get

$$
\begin{aligned}
A\left(Q_{1}, \ldots, Q_{t} ; \delta\right) & \leq \sum_{\bar{n}_{i} \in Q_{i}} S\left(\bar{n}_{1}, \ldots, \bar{n}_{t}\right) \\
& \ll\left(N^{t \theta_{t}}+\delta N^{t(k-1) / k} \log ^{t-1} N\right)\left|Q_{1}\right| \ldots\left|Q_{t}\right| .
\end{aligned}
$$

This completes the proof of Lemma 3 .

Lemma 4. Suppose $k_{1}, k_{2}, N_{1}, N_{2}$ are natural numbers, $\delta>0$, and $Q_{1}$ and $Q_{2}$ are subsets of $\left\{N_{1}, \ldots, 2 N_{1}\right\}$ and $\left\{N_{2}, \ldots, 2 N_{2}\right\}$ respectively. Let $A$ denote the number of solutions to the inequality

$$
\left|n_{1}^{1 / k_{1}} n_{2}^{1 / k_{2}}-\bar{n}_{1}^{1 / k_{1}} \bar{n}_{2}^{1 / k_{2}}\right| \leq \delta, \quad n_{1}, \bar{n}_{1} \in Q_{1}, n_{2}, \bar{n}_{2} \in Q_{2} .
$$

Then

$$
A \ll N_{1} N_{2} \log N_{2}+\delta N_{1}^{2-1 / k_{1}} N_{2}^{2-1 / k_{2}} .
$$

Proof. The idea of the proof of Lemma 4 comes from [2]. Given $r \leq 2 N_{1}$ and $s \leq 2 N_{2}$ let $V_{r s}$ stand for the number of solutions to

$$
\left|n_{1}^{1 / k_{1}} n_{2}^{1 / k_{2}}-\bar{n}_{1}^{1 / k_{1}} \bar{n}_{2}^{1 / k_{2}}\right| \leq \delta
$$

in $n_{1}, \bar{n}_{1} \in Q_{1}, n_{2}, \bar{n}_{2} \in Q_{2}$ such that $\left(n_{1}, \bar{n}_{1}\right)=r$ and $\left(n_{2}, \bar{n}_{2}\right)=s$.

By (10) we get

$$
\left|\frac{n_{1}}{\bar{n}_{1}}-\left(\frac{\bar{n}_{2}}{n_{2}}\right)^{k_{1} / k_{2}}\right| \ll \delta N_{1}^{-1 / k_{1}} N_{2}^{-1 / k_{2}} .
$$

Since the points $n_{1} / \bar{n}_{1}$ are $\left(\frac{r}{2 N_{1}}\right)^{2}$-spaced, we get

$$
V_{r s} \ll\left(1+\delta N_{1}^{-1 / k_{1}} N_{2}^{-1 / k_{2}} N_{1}^{2} r^{-2}\right) T_{s}^{2}
$$

by the Dirichlet box principle, where

$$
T_{s}=\left|\left\{n_{2} \in Q_{2} \mid n_{2} \equiv 0(\bmod s)\right\}\right| \ll N_{2} / s .
$$

Thus we obtain

$$
V_{r s} \ll N_{2}^{2} s^{-2}+\delta N_{1}^{2-1 / k_{1}} N_{2}^{2-1 / k_{2}} r^{-2} s^{-2} .
$$

Similarly, we have

$$
V_{r s} \ll N_{1}^{2} r^{-2}+\delta N_{1}^{2-1 / k_{1}} N_{2}^{2-1 / k_{2}} r^{-2} s^{-2} .
$$

So

$$
V_{r s} \ll \min \left(N_{2}^{2} / s^{2}, N_{1}^{2} / r^{2}\right)+\delta N_{1}^{2-1 / k_{1}} N_{2}^{2-1 / k_{2}} r^{-2} s^{-2} .
$$

Summing over $r$ and $s$ we complete the proof. 
3. Proof of Theorem $1(k \geq 4)$. It is easy to check that

$$
\left[n^{1 / k}+\Delta\right]-\left[n^{1 / k}-\Delta\right]= \begin{cases}1, & \left\|n^{1 / k}\right\| \leq \Delta, \\ 0, & \text { otherwise }\end{cases}
$$

So we have

$$
\text { (16) } \begin{aligned}
S_{k} & =\sum_{n_{i} \in G_{i}}\left(\left[\left(n_{1} \ldots n_{k}\right)^{1 / k}+\Delta\right]-\left[\left(n_{1} \ldots n_{k}\right)^{1 / k}-\Delta\right]\right) \\
& =2 \Delta T_{k}+\sum_{n_{i} \in G_{i}}\left(b\left(\left(n_{1} \ldots n_{k}\right)^{1 / k}-\Delta\right)-b\left(\left(n_{1} \ldots n_{k}\right)^{1 / k}+\Delta\right)\right) .
\end{aligned}
$$

It is well known that

$$
b(t)=-\sum_{0<|h| \leq H} \frac{e(h t)}{2 \pi i h}+O\left(\min \left(1, \frac{1}{H\|t\|}\right)\right)
$$

and

$$
\min \left(1, \frac{1}{H\|t\|}\right)=\sum_{h=-\infty}^{\infty} a_{h} e(h t)
$$

with

$$
a_{0} \ll \frac{\log H}{H}, \quad a_{h} \ll \min \left(\frac{1}{|h|}, \frac{H}{h^{2}}\right) \quad \text { if } h \neq 0 .
$$

Using (17)-(19), we have

$$
\begin{aligned}
\sum_{n_{i} \in G_{i}} b\left(\left(n_{1} \ldots n_{k}\right)^{1 / k} \pm \Delta\right) & \\
= & -\sum_{0<|h| \leq H} \frac{1}{2 \pi i h} \sum_{n_{i} \in G_{i}} e\left(h\left(n_{1} \ldots n_{k}\right)^{1 / k} \pm h \Delta\right) \\
& +O\left(\sum_{n_{i} \in G_{i}} \min \left(1, \frac{1}{H\left\|\left(n_{1} \ldots n_{k}\right)^{1 / k} \pm \Delta\right\|}\right)\right) \\
\ll & \frac{T_{k} \log H}{H}+\sum_{h=1}^{\infty} \min \left(\frac{1}{h}, \frac{H}{h^{2}}\right)\left|\sum_{n_{i} \in G_{i}} e\left(h\left(n_{1} \ldots n_{k}\right)^{1 / k}\right)\right|
\end{aligned}
$$

So the problem is now reduced to the estimation of the exponential sum

$$
\Sigma(h)=\sum_{n_{i} \in G_{i}} e\left(h\left(n_{1} \ldots n_{k}\right)^{1 / k}\right) .
$$

Now suppose $k \geq 4$ and $t=[k / 2]$, then $t \geq 2$. Applying Lemma 1 to the sequences $A=\left\{h\left(n_{1} \ldots n_{t}\right)^{1 / k} \mid n_{i} \in G_{i}, i=1, \ldots, t\right\}$ and $B=$ $\left\{\left(n_{t+1} \ldots n_{k}\right)^{1 / k} \mid n_{i} \in G_{i}, i=t+1, \ldots, k\right\}$, we get

$$
\Sigma(h) \ll\left(h N V_{1} V_{2}\right)^{1 / 2},
$$


where $V_{1}=A\left(G_{1}, \ldots, G_{t} ; 2^{-1}(2 N)^{-(k-t) / k} h^{-1}\right)$ and $V_{2}=A\left(G_{t+1}, \ldots, G_{k}\right.$; $\left.2^{-1}(2 N)^{-t / k} h^{-1}\right) . V_{1}$ and $V_{2}$ can then be estimated by Lemma 3 . We have

$$
V_{1} \ll\left(N^{t \theta_{t}}+h^{-1} N^{t-1} \log ^{t-1} N\right)\left|G_{1}\right| \ldots\left|G_{t}\right|
$$

and

$$
V_{2} \ll\left(N^{(k-t) \theta_{k-t}}+h^{-1} N^{k-t-1} \log ^{k-t-1} N\right)\left|G_{t+1}\right| \ldots\left|G_{k}\right| .
$$

Combining (20)-(23), we get

$$
\begin{aligned}
& \sum_{n_{i} \in G_{i}} b\left(\left(n_{1} \ldots n_{k}\right)^{1 / k} \pm \Delta\right) \\
\ll & \frac{T_{k} \log H}{H}+H^{1 / 2} T_{k}^{1 / 2} N^{\left(1+t \theta_{t}+(k-t) \theta_{k-t}\right) / 2}+T_{k}^{1 / 2} N^{(k-1) / 2} \log \left({ }^{(k-2) / 2} N .\right.
\end{aligned}
$$

Choosing $H$ such that the first two terms in (24) are equal, we get

$$
\begin{aligned}
& \sum_{n_{i} \in G_{i}} b\left(\left(n_{1} \ldots n_{k}\right)^{1 / k} \pm \Delta\right) \\
& \ll T_{k}^{2 / 3} N^{\left(1+t \theta_{t}+(k-t) \theta_{k-t}\right) / 3} \log ^{1 / 3} N+T_{k}^{1 / 2} N^{(k-1) / 2} \log ^{(k-2) / 2} N \\
& \ll T_{k}^{1 / 2} N^{(k-1) / 2} \log ^{(k-2) / 2} N \text {. }
\end{aligned}
$$

Hence Theorem 1 for the case $k \geq 4$ follows from (16) and (25).

4. Proof of Theorem $1(k=3)$. Choosing $H=N / \log N$, we have

$$
\begin{aligned}
\sum_{n_{i} \in G_{i}} b\left(\left(n_{1} n_{2} n_{3}\right)^{1 / 3} \pm \Delta\right) & \\
= & -\sum_{0<|h| \leq H} \frac{1}{2 \pi i h} \sum_{n_{i} \in G_{i}} e\left(h\left(n_{1} n_{2} n_{3}\right)^{1 / 3} \pm h \Delta\right) \\
& +O\left(\sum_{n_{i} \in G_{i}} \min \left(1, \frac{1}{H\left\|\left(n_{1} n_{2} n_{3}\right)^{1 / 3} \pm \Delta\right\|}\right)\right) \\
\ll & \frac{T_{3} \log H}{H}+S_{1}+S_{2},
\end{aligned}
$$

where

$$
S_{1}=\left|\sum_{h \leq H} \frac{1}{h} \sum_{n_{i} \in G_{i}} e\left(h\left(n_{1} n_{2} n_{3}\right)^{1 / 3} \pm h \Delta\right)\right|
$$

and

$$
S_{2}=\left|\sum_{h \leq H^{2}} a_{h} \sum_{n_{i} \in G_{i}} e\left(h\left(n_{1} n_{2} n_{3}\right)^{1 / 3} \pm h \Delta\right)\right| .
$$

We only estimate $S_{2}$ and we can estimate $S_{1}$ in the same way. 
We have

$$
\begin{aligned}
\sum_{h \leq H^{2}} a_{h} \sum_{n_{i} \in G_{i}} e\left(h\left(n_{1} n_{2} n_{3}\right)^{1 / 3} \pm h \Delta\right) \\
=\sum_{L=2^{l} \geq 1} \sum_{\substack{L \leq h<2 L \\
n_{i} \in G_{i}}} a_{h} e\left(h\left(n_{1} n_{2} n_{3}\right)^{1 / 3} \pm h \Delta\right) \\
\ll \sum_{L=2^{l}} c(L)\left|\sum_{\substack{L \leq h<2 L \\
n_{i} \in G_{i}}} \frac{a_{h} e( \pm h \Delta)}{c(L)} e\left(h\left(n_{1} n_{2} n_{3}\right)^{1 / 3}\right)\right|,
\end{aligned}
$$

where $c(h)=\min \left(1 / h, H / h^{2}\right)$.

Now we only need to estimate

$$
\Sigma(L)=\sum_{\substack{L \leq h<2 L \\ n_{i} \in G_{i}}} \frac{a_{h} e( \pm h \Delta)}{c(L)} e\left(h\left(n_{1} n_{2} n_{3}\right)^{1 / 3}\right) .
$$

Applying Lemma 1 to the sequences $A=\left\{h n_{1}^{1 / 3} \mid L \leq h<2 L, n_{1} \in G_{1}\right\}$ and $B=\left\{\left(n_{2} n_{3}\right)^{1 / 3} \mid n_{2} \in G_{2}, n_{3} \in G_{3}\right\}$, we get

$$
\Sigma(L) \ll\left(L N V_{3} V_{4}\right)^{1 / 2},
$$

where

$$
V_{3}=\sum_{\substack{x, x^{\prime} \in A \\ 2(2 N)^{2 / 3}\left|x-x^{\prime}\right| \leq 1}} 1 \text { and } V_{4}=\sum_{\substack{y, y^{\prime} \in B \\ 2 L(2 N)^{1 / 3}\left|y-y^{\prime}\right| \leq 1}} 1 .
$$

By Lemma 4 we have

$$
V_{3} \ll L N \log N
$$

and

$$
V_{4} \ll N^{2} \log N+N^{3} / L .
$$

Combining (27)-(30), we obtain

$$
S_{2} \ll N^{5 / 2} \log ^{1 / 2} N
$$

Similarly,

$$
S_{1} \ll N^{5 / 2} \log ^{1 / 2} N
$$

Hence Theorem 1 for the case $k=3$ follows from (16), (26), (31) and (32).

Acknowledgements. The author would like to thank the referee for his valuable comments and Prof. H. Iwaniec for his encouragement and help. 


\section{References}

[1] E. Fouvry and H. Iwaniec, Exponential sums with monomials, J. Number Theory 33 (1989), 311-333.

[2] H. Iwaniec and A. Sárközy, On a multiplicative hybrid problem, ibid. 26 (1987), 89-95.

[3] E. C. Titchmarsh, The Theory of the Riemann Zeta-function, 2nd ed. (revised by D. R. Heath-Brown), Cambridge Univ. Press, Oxford, 1986.

DEPARTMENT OF MATHEMATICS

SHANDONG UNIVERSITY

JINAN 250100, CHINA

Received on 22.10.1993

and in revised form on 25.5.1994 and 13.10.1994 